
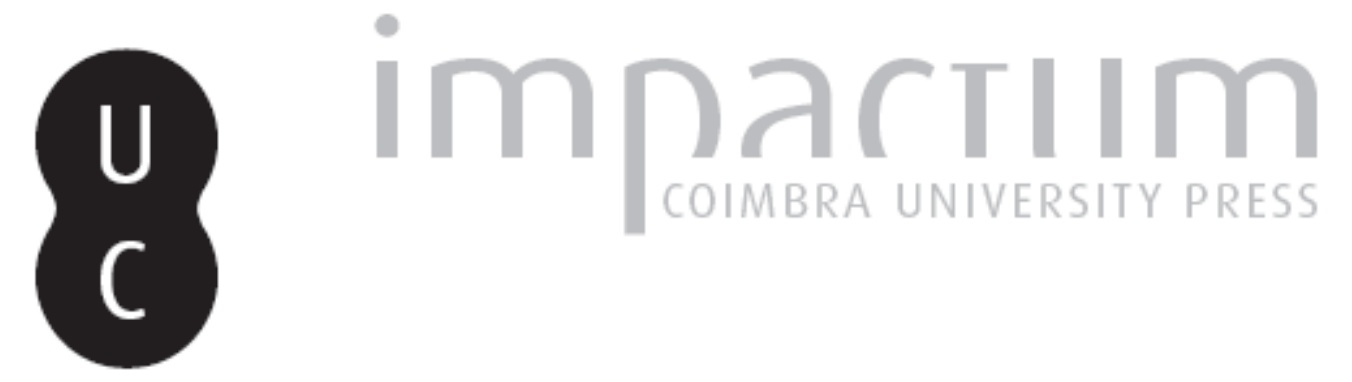

\title{
Ontologia e filosofia del linguaggio in Platone: acluni studi recenti
}

\section{Autor(es): $\quad$ Fronterotta, Francesco}

Publicado por: Imprensa da Universidade de Coimbra

URL persistente:

URl:http://hdl.handle.net/10316.2/42203

DOI:

DOl:https://doi.org/10.14195/2183-4105_9_5

Accessed : $\quad$ 26-Apr-2023 08:36:19

A navegação consulta e descarregamento dos títulos inseridos nas Bibliotecas Digitais UC Digitalis, UC Pombalina e UC Impactum, pressupõem a aceitação plena e sem reservas dos Termos e Condições de Uso destas Bibliotecas Digitais, disponíveis em https://digitalis.uc.pt/pt-pt/termos.

Conforme exposto nos referidos Termos e Condições de Uso, o descarregamento de títulos de acesso restrito requer uma licença válida de autorização devendo o utilizador aceder ao(s) documento(s) a partir de um endereço de IP da instituição detentora da supramencionada licença.

Ao utilizador é apenas permitido o descarregamento para uso pessoal, pelo que o emprego do(s) título(s) descarregado(s) para outro fim, designadamente comercial, carece de autorização do respetivo autor ou editor da obra.

Na medida em que todas as obras da UC Digitalis se encontram protegidas pelo Código do Direito de Autor e Direitos Conexos e demais legislação aplicável, toda a cópia, parcial ou total, deste documento, nos casos em que é legalmente admitida, deverá conter ou fazer-se acompanhar por este aviso.

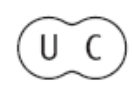




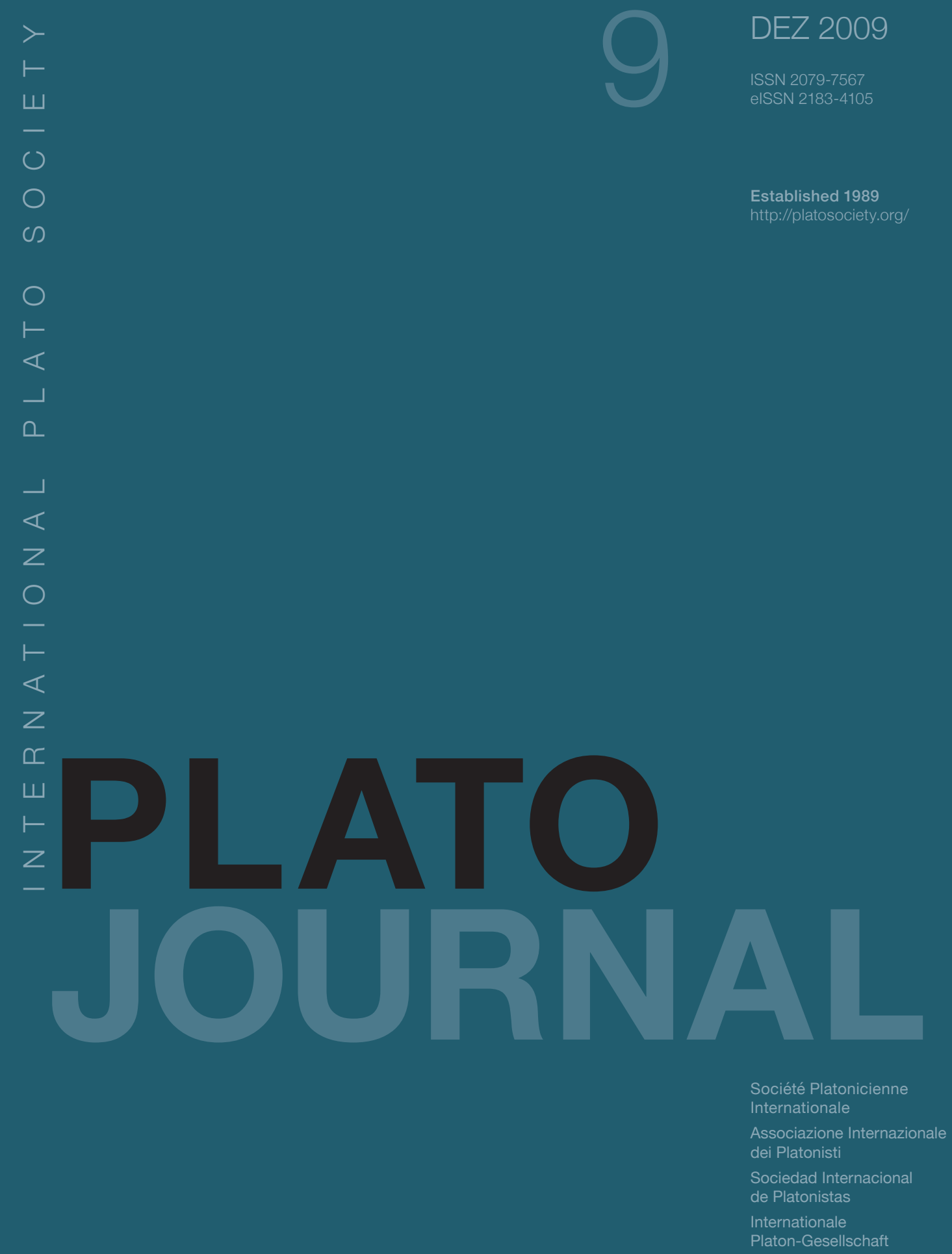




\section{ONTOlogia E FILOSOFIA DEL LINGUAGgio IN PLATONE}

\section{AlCUni STUdi RECENTI}

Presento in questa rassegna, certamente non esaustiva né quantitativamente né qualitativamente, alcuni studi su Platone, comparsi prevalentemente, ma non esclusivamente, negli ultimi cinque anni, sul tema dell'ontologia e della filosofia del linguaggio. Trattandosi di una rubrica che adotterà una cadenza regolare, verosimilmente triennale, è opportuno fornire in questa prima uscita alcune indicazioni di carattere generale. Innanzitutto, l'etichetta adottata, tanto in relazione all'ontologia' quanto alla 'filosofia del linguaggio', fa riferimento a categorie appartenenti piuttosto al pensiero filosofico e scientifico moderno e contemporaneo che non alla riflessione classica: parlando di 'ontologia' di Platone, si allude pertanto, in termini generali, alla concezione platonica dell'essere e di ciò

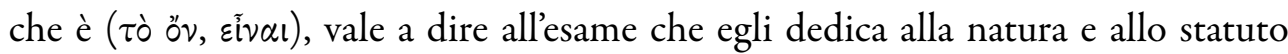
delle cose che sono; in quanto poi, come si sa, Platone distingue fra un ambito di oggetti di natura e statuto sensibile e un ambito di oggetti di natura e statuto intellegibile, di cui viene talora precisato in modo esplicito che si situano su un piano di realtà distinto e separato dal mondo fisico, tale ontologia implica una serie di sviluppi di carattere metafisico. Analogamente, per quanto riguarda la 'filosofia del linguaggio', non soltanto verranno presi in considerazione i contributi relativi all'indagine intorno alla concezione platonica del linguaggio, ma l'attenzione sarà rivolta in modo particolare al caratteristico approccio, che Platone condivide con alcuni dei suoi predecessori e successori, alla questione specifica della relazione fra i nomi e le cose, vale a dire alle modalità operative $\mathrm{e}$ funzionali, naturali o convenzionali, del linguaggio rispetto agli oggetti di cui parla, in una prospettiva che, nella filosofia contemporanea, specie dopo le opere dell'ultimo Wittgenstein, ha perduto gran parte del suo interesse.

Fatte queste precisazioni, occorre ancora preliminarmente rilevare, come è ben noto agli studiosi e ai lettori di Platone, che le tematiche individuate si

PLATO, The electronic Journal of the International Plato Society, n 9, 2009.

http://gramata.univ-paris1.fr/Plato

(c) All rights of reproduction of any form reserved. 
trovano declinate, negli scritti del filosofo, in modo assai peculiare. Facendo riferimento, per esempio, a un passo del Cratilo (439b10-440c1), appare che, nel momento più acuto di una polemica nei confronti della dottrina eraclitea, Socrate, che conduce la discussione, introduce l'argomento seguente: se tutte le cose sono in movimento e mutano eternamente, come pretendono Eraclito e i suoi seguaci, nessuno potrà conoscere alcunché. Infatti, ciò che non è mai identico a sé non può essere 'in sé' ( $\kappa \alpha \theta^{\prime}$ ' $\left.\tau \tau o ́\right)$ né può essere un 'qualcosa' $(\tau \iota)$ determinato; al contrario, se rimane identicamente nella propria condizione, senza allontanarsi dalla propria

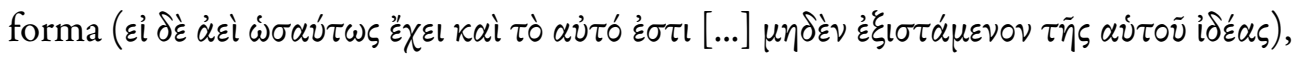
bisognerà ammettere che non è soggetto al mutamento e al movimento (<oj̉k $\tilde{\alpha}^{2}$

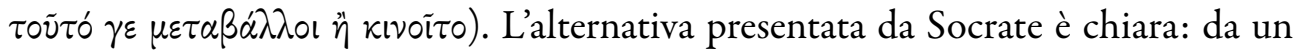
lato, la dottrina eraclitea del perenne divenire, che riconosce il flusso inarrestabile di tutte le cose e, così facendo, nega la possibilità che esistano enti 'permanenti' in sé stessi e perciò realmente essenti e in possesso di caratteristiche determinate e stabili; dall'altro, in una qualche sua forma, la 'teoria delle idee', che prevede l'esistenza di certe entità immutabili e auto-identiche diverse dalla realtà sensibile e poste al di là di questa. Già a questo livello, l'analisi ha rivelato i paradossi impliciti nella posizione eraclitea, ma non è stato ancora introdotto l'argomento decisivo (439e7-440c3): se tutto scorre eternamente e muta, nulla sarà uguale a sé stesso né

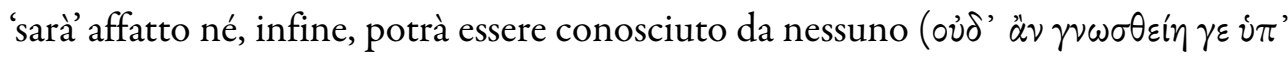

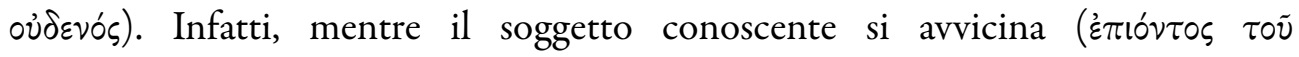
$\gamma \nu \omega \sigma o \mu \varepsilon ́ v 0 v)$, l'oggetto conosciuto modificherà la propria condizione e, reso così

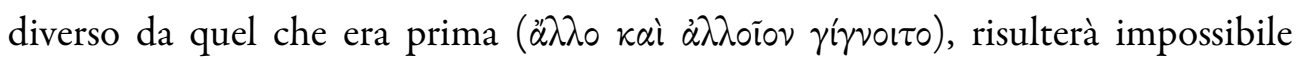

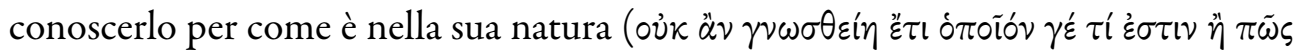
है (ov). In un analogo contesto teorico, alla conclusione del libro $\mathrm{V}$ della Repubblica (476d5-477b11; 477e8-480a13), Socrate svolge un'articolata dimostrazione per definire lo statuto epistemologico della vera conoscenza dei filosofi. Chi conosce,

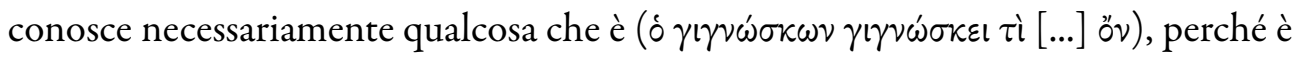

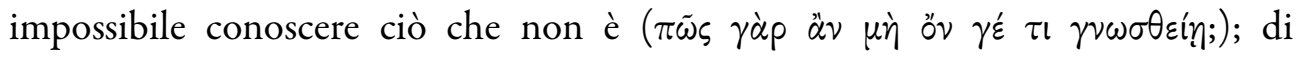
conseguenza, è possibile formulare il seguente principio: 'ciò che è assolutamente,

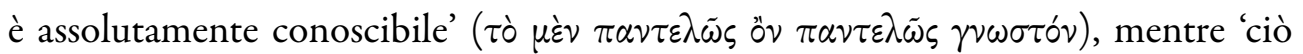

PLATO, The electronic Journal of the International Plato Society, n 9, 2009.

http://gramata.univ-paris1.fr/Plato

(c) All rights of reproduction of any form reserved. 
che non è assolutamente, è assolutamente inconoscibile' ( $\mu \grave{\eta}$ öv $\delta \grave{\varepsilon} \mu \eta \delta \alpha \mu \tilde{\eta} \pi \dot{\alpha} \nu \tau \eta$ $\ddot{\alpha} \gamma \nu \omega \sigma \tau \circ v)$. Infatti, in quanto implica una facoltà cognitiva diversa, ogni forma di conoscenza ha un oggetto specifico e diverso. La vera conoscenza, la scienza, posta al culmine della gerarchia, conosce l'essere per come è; l'ignoranza, invece, assenza di ogni conoscenza, ignora totalmente ciò che non è affatto (tralascio ovviamente, in questo contesto, ogni ulteriore difficoltà connessa all'interpretazione di questi passi, che sono stati e sono al centro di un dibattito sterminato). Questi argomenti esemplari del Cratilo e della Repubblica manifestano abbastanza chiaramente, mi pare, il caratteristico nesso teorico che è posto da Platone fra la sua prospettiva ontologica (relativa alla natura e allo statuto delle cose che sono), la dottrina epistemologica che ne deriva e ne dipende (relativa alla 'conoscibilită dei diversi oggetti in virtù del loro diverso statuto ontologico) e la conseguente concezione del linguaggio come strumento semantico per la designazione e la 'significazione' delle cose che sono e delle loro relazioni (ancora una volta in base alla diversa tipologia delle cose che sono e al loro diverso statuto ontologico). Si comprenderà allora come, nella presente rassegna, si collochi innanzitutto al centro dell'attenzione, comunque la si intenda, (A) la 'teoria delle idee', nell'ambito della quale si trovano intimamente coniugati aspetti ed elementi ontologici, epistemologici e linguistici, benché, naturalmente, si diano anche casi, meno numerosi, di (B) studi dedicati a questi temi nella loro specificità ed esclusività, per esempio all'ontologia, nel senso analitico di uno screening delle cose esistenti e della loro struttura materiale, con riferimento al rapporto tutto-parti, unitàmolteplicità e così via, oppure alla filosofia del linguaggio concepita come una teoria della proposizione e della verità proposizionale.

Prendendo le mosse dalla categoria (A), si può trarre spunto da alcuni volumi che hanno ricollocato la teoria delle idee, anche criticamente, al centro dell'analisi. Ricordo innanzitutto, più per il suo carattere di sintesi esegetica e dottrinale che non per elementi di particolare originalità, il volume di R.M. Dancy, Plato's Introduction of Forms (Cambridge, 2004), che ha il merito di riproporre ordinatamente un'immagine tradizionale, e ampiamente diffusa negli

PLATO, The electronic Journal of the International Plato Society, n 9, 2009.

http://gramata.univ-paris1.fr/Plato

(c) All rights of reproduction of any form reserved. 
studi anglo-americani (di Ross, Allen e Vlastos, per esempio), dell'evoluzione della riflessione platonica, dai dialoghi giovanili, che farebbero emergere l'eredità metodologica dell'indagine socratica, alle opere della maturità, che, fin dal Menone, manifesterebbero invece la classica tendenza metafisica che conduce alla postulazione di certe entità separate, eterne e immutabili, di cui dialoghi come il Fedone e il Simposio preciserebbero via via lo statuto e la funzione ontologici. Nel contesto di questa ricostruzione classica si inseriscono due volumi miscellanei, il primo dei quali, in lingua italiana, raccoglie una serie di articoli di più autori, ma tutti incentrati sulle diverse implicazioni che le nozioni di eidos e di idea comportano negli scritti platonici, nel dibattito della prima Accademia e nel corso della tradizione platonica (F. Fronterotta \& W. Leszl, eds., Eidos-Idea. Platone, Aristotele e la tradizione platonica, Sankt Augustin, 2005); mentre il secondo riunisce alcuni studi di G. Fine, già precedentemente editi, che sono per lo più dedicati alla teoria platonica delle idee e alle conseguenza che essa determina sul piano epistemologico (G. Fine, Plato on knowledge and forms. Selected Essays, Oxford, 2003; essenzialmente finalizzata a una riedizione di saggi ormai classici negli studi platonici anglo-americani del XX secolo, con l'aggiunta di pochi lavori più recenti, è invece la raccolta, curata dalla stessa Fine, dal titolo Plato I: Metaphysics and Epistemology, New York 2000, con articoli di G. Vlastos, D. Scott, J.L. Ackrill, T.H. Irwin, A. Nehamas, D.T. Devereux, G. Fine, G. Santas, S.M. Cohen, G.E.L. Owen, M.F. Burnyeat, J.M. Cooper, M. Frede, J. McDowell, S.K. Strange e L. Brown).

Per quanto riguarda il primo dei volumi citati, esso intende contrapporsi alla tendenza esegetica, assai netta nell'ultimo cinquantennio negli studi platonici, che ha rivolto prevalentemente la propria attenzione all'indagine di problemi specifici o di singoli aspetti dell'opera di Platone, perdendo talvolta di vista l'orizzonte concettuale e lo sfondo filosofico entro il quale bisogna collocare il suo pensiero e che pare costituire il nucleo teorico della riflessione del filosofo ateniese (p. xxix).

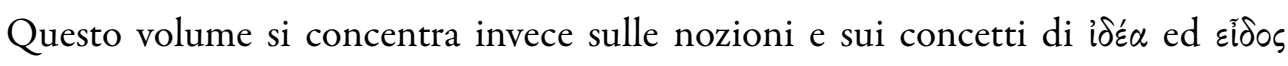

PLATO, The electronic Journal of the International Plato Society, n 9, 2009. http://gramata.univ-paris1.fr/Plato (c) All rights of reproduction of any form reserved. 
nella filosofia di Platone e nel dibattito che le dottrine platoniche hanno suscitato nella tradizione posteriore, a partire dalla prima Accademia e da Aristotele, fino a giungere a tracciare un quadro schematico e sintetico delle principali correnti platonizzanti del pensiero antico. Il comune denominatore teorico condiviso dai partecipanti al volume consiste propriamente, al di là delle possibili, e ovvie, divergenze nell'interpretazione di singole questioni o delle diversità di accento e di interesse su questo o su quello spunto teoretico, nella convinzione che

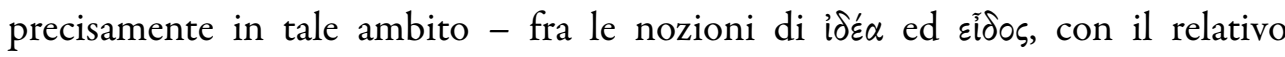
apparato concettuale che si estende dall'ontologia alla metafisica e si costituisce fra la metodologia dialettica e l'epistemologia - si situa almeno una, se non la principale, linea di sviluppo della riflessione di Platone, per reagire fra l'altro alla tendenza di quanti fra gli studiosi, per provocazione, per moda o per semplice 'stanchezza' esegetica, hanno contribuito a svalutare il ruolo e l'importanza delle teoria delle idee, finendo così, volontariamente oppure no, per spingere la filosofia di Platone verso (storicamente) impropri esiti scettici. Indubbiamente convergenti, su questo piano, sono i contributi di M. Baltes e M.-L. Lakmann (pp. 1-23, che produce un esame dei termini e dei concetti fondamentali della teoria delle idee); di L. Brisson e di J.-F. Pradeau (pp. 25-36 e 75-89, che affrontano rispettivamente il problema della partecipazione delle cose sensibili alle idee e la

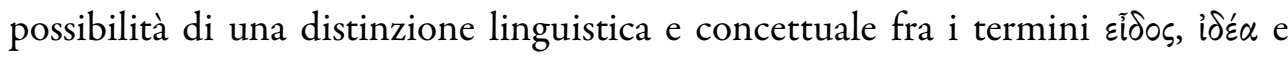

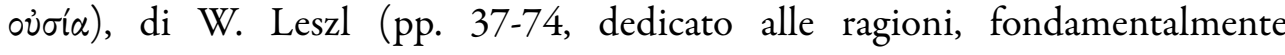
epistemologiche, che hanno indotto Platone a porre l'esistenza delle idee come entità distinte e separate dal mondo sensibile) e di G. Sillitti e di D. O’Brien (pp. 91-101 e 115-59, che indagano la questione della natura e della collocazione di due fra le idee che sembrano possedere, nella descrizione platonica, uno statuto e dei requisiti del tutto particolari: l'idea del bene nella Repubblica e l'idea del diverso, e del non essere, nel Sofista). Fra gli obiettivi di questo volume va valorizzato pertanto, in prima battuta, quello di confrontarsi con la linea esegetica, propugnata per esempio da F.J. Gonzalez (The third way: new directions in Platonic studies, Lahnam, 1995; Plato's dialectic of forms, in Plato's forms.

PLATO, The electronic Journal of the International Plato Society, n 9, 2009.

http://gramata.univ-paris1.fr/Plato

(c) All rights of reproduction of any form reserved. 
Varieties of interpretation, ed. by W.A. Welton, Lanham, 2002), secondo la quale non esisterebbe, nella riflessione di Platone, una 'teoria delle idee', perché i dialoghi non solo non sarebbero in grado di fornire nessuna precisazione relativa alla natura e allo statuto delle idee, alla loro funzione e al loro rapporto con il mondo sensibile, ma neanche rivelerebbero un reale interesse e un'intenzione consapevole di rispondervi. I saggi sopra citati costituiscono in effetti un tentativo di rispondere punto per punto a questi rilievi.

La raccolta di G. Fine presenta a sua volta, pur nella varietà dei temi affrontati nei diversi saggi, una coerenza d'insieme specie rispetto a due questioni centrali: la distinzione epistemica fra opinione e scienza (pp. 44-116 e 225-51) e il problema della separazione delle idee, anche attraverso l'analisi della critica aristotelica a Platone (pp. 252-425). Sulla prima questione, Fine ripropone la sua nota tesi, seconda la quale la vera conoscenza, la scienza, va concepita come una forma di opinione vera di cui si sia in grado di rendere conto attraverso una giustificazione o una spiegazione, capaci di 'fissare' la verità di un'opinione conferendole i tratti stabili e immutabili della scienza, dunque nella forma di un justified true belief. Nonostante il ricorso di Platone, in non pochi passi dei dialoghi, a una distinzione di carattere ontologico, che fonda la superiorità della scienza sull'opinione attraverso la distinzione di oggetti (intellegibili o sensibili) che ciascuna di esse assume come proprio contenuto, alcuni passi degli scritti platonici (particolarmente nel Menone, nei libri centrali della Repubblica e nel Teeteto) indurrebbero a ritenere che la verità del pensiero e del discorso non è in relazione con la realtà o la natura degli oggetti pensati o detti, perché sarebbe basata nel contesto proposizionale del giudizio che la esprime. Conoscenza, o scienza, e opinione finirebbero perciò per coincidere l'una con l'altra, salvo che la prima implicherebbe un 'sovrappiù di cui la seconda sarebbe priva, un 'sovrappiù' consistente in fin dei conti appunto nella giustificazione o nella spiegazione causale che permette non solo di formulare un giudizio, ma anche di renderne conto, cioè di manifestarne le ragioni o le cause. Rispetto al problema della

PLATO, The electronic Journal of the International Plato Society, n 9, 2009.

http://gramata.univ-paris1.fr/Plato

(c) All rights of reproduction of any form reserved. 
separazione delle idee, Fine si chiede soprattutto se essa vada intesa nel senso 'locale' di una scansione fra due mondi separati, come Aristotele, per fini polemici, pare concepirla, oppure come l'indicazione della sussistenza o dell'esistenza autonoma e indipendente delle idee di fronte alle cose sensibili. In questo secondo significato, che Fine predilige, l'affermazione della separazione delle idee non sembra implicare necessariamente delle conseguenze sul piano metafisico, quanto piuttosto sul piano logico dei significati, nella misura in cui le idee si configurerebbero non tanto come delle entità determinate appunto dal punto di vista metafisico, quanto piuttosto, secondo un'interpretazione già predentemente diffusa nel mondo anglo-sassone, come unità di significato o come criteri standard per la distinzione e la definizione delle cose sensibili che ne partecipano, tale partecipazione dovendosi perciò intendere come una relazione di carattere eminentemente logico. Si possono inoltre ricordare in proposito, per un esame degli aspetti propriamente logico-metefisici dello statuto delle idee platoniche, tre volumi recenti, di A. Silverman, The Dialectic of Essence: A Study of Plato's Metaphysics, Princeton, 2003, di B. Strobel, 'Dieses' und 'So etwas'. Zur ontologischer Klassifikation platonischer Formen, Göttingen, 2007, e di F.A. Grabowski, Plato, metaphysics and the Forms, London-New York, 2008. Particolarmente il secondo di essi distingue accuratamente fra una concezione delle idee come oggetti o entità individuali e come generi o tipi che si determinano in base al carattere dell'universalità, passando in rassegna i problemi dell'auto-predicazione delle idee, delle idee dei termini relativi, della separazione fra sensibile e intellegibile e della loro reciproca relazione, attraverso una lettura piuttosto approfondita dei passi pertinenti dei dialoghi e un'analisi della letteratura critica più influente (1).

Un altro problema connesso all'interpretazione della teoria delle idee e delle sue implicazioni epistemologiche e logico-linguistiche è quello, non nuovo, ma ravvivato in anni recenti, della presenza di questa teoria fin nei dialoghi giovanili, o 'socratici', di Platone. Non pochi lavori dell'ultimo decennio toccano la

PLATO, The electronic Journal of the International Plato Society, n 9, 2009.

http://gramata.univ-paris1.fr/Plato

(c) All rights of reproduction of any form reserved. 
questione (2), ma mi soffermerò esclusivamente qui su una serie di articoli che D. Wolfsdorf ha dedicato alla natura e alla funzione causale delle idee nei primi dialoghi di Platone (3), i cui esiti sono in qualche modo riassunti, o piuttosto realizzati compiutamente, nel suo volume Trials of Reason: Plato and the Crafting of Philosophy (New York, 2008). Che vi sia una versione della 'teorie delle idee' fin nei primi dialoghi platonici è tesi che fu difesa già, con argomenti convincenti, in un celebre saggio di R.E. Allen sull'Eutifrone (Plato's Euthyphro and the earlier theory of forms, London, 1970); lo stesso Allen, tuttavia, segnalava le evidenti differenze che si pongono fra la 'teoria delle idee' dei primi dialoghi da quella che si trova nei dialoghi della maturità di Platone, con la ben nota caratterizzazione metafisica della separazione fra le idee e le cose sensibili, che si amplifica fino a configurare due mondi distinti e appunto separati dell'essere e del divenire. La strategia adottata negli studi più recenti su questo tema, e ben esemplificata dal lavoro di Wolfsdorf, consiste nel prestare particolare attenzione a due aspetti specifici: quello della 'causalità' o dell'azione 'causale' delle idee nei primi dialoghi e quello del loro ruolo e della loro collocazione come plausibile risposta all'interrogazione socratica del ' $\tau$ í $̇$ $\sigma \tau$ ' negli stessi dialoghi. In entrambi i casi, e seguendo entrambi i percorsi, si giunge a una descrizione dell' $\varepsilon \tilde{\delta} \delta \circ \varsigma$, o dell' $i \delta \varepsilon ́ \alpha$, cui si attribuisce, per un verso, il carattere dell'universalità (giacché l'idea è indicata, per esempio nell'Ippia maggiore e nell'Eutifrone, come la causa unica e ultima della bellezza o della santità di tutte le possibili cose o situazioni belle e sante, che di quella causa sono altrettanti effetti, nella forma di esemplificazioni o istanziazioni particolari, che si raccolgono sotto la sua definizione generale) e, per altro verso, il carattere dell'individualità o di una qualche sorta di sussistenza autonoma (nella misura in cui l' $\varepsilon \tilde{\delta} \delta \circ \varsigma$, o l' 'i $\delta \varepsilon ́ \alpha$, costituiscono l'unica risposta corretta alla domanda socratica del ' $\tau$ ' $̇$ $\sigma \tau \imath$ ', che tende a identificare un definiens che coincida pienamente con un definiendum ben distinto da tutte le sue applicazioni specifiche). Ne deriva che, tanto dal punto di vista logico-epistemologico della sua apprensione ed

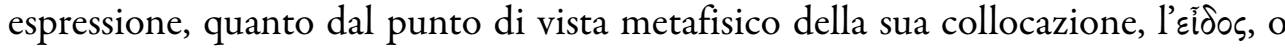

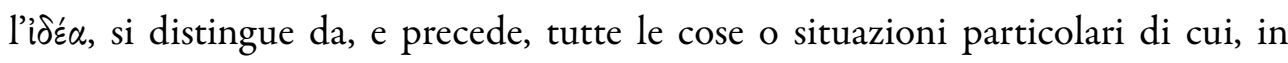

PLATO, The electronic Journal of the International Plato Society, n 9, 2009.

http://gramata.univ-paris1.fr/Plato

(c) All rights of reproduction of any form reserved. 
qualità di causa, fornisce altresì il concetto e la definizione, il che conduce alla conclusione, tuttora in discussione nel dibattito critico, secondo la quale, fin nei dialoghi platonici giovanili, le idee esistono indipendentemente dall'atto logico e intellettuale della definizione e della conoscenza da parte di un soggetto, dotate perciò di un'esistenza distinta, autonoma e prioritaria rispetto alle cose molteplici e particolari di cui pure costituiscono l'essenza.

Nell'ambito della sfera eidetica, così costituita, un'ampia discussione continua a sussistere intorno allo statuto di alcune idee in particolare. Mi riferisco naturalmente, e in primo luogo, al caso dell'idea del bene nella Repubblica che, oltre a rappresentare uno dei problemi più controversi nella tradizione platonica antica, ha ricevuto di recente nuove attenzioni. Che sia esaminata di per sé e rispetto alla sua collocazione fra le altre idee e alla sua funzione in relazione a esse (si vedano in proposito gli studi raccolti nel volume di G. Reale and S. Scolnicov, eds., New Images of Plato. The Idea of the Good, Sankt Augustin, 2002) oppure come punto focale verso il quale tende la stessa struttura teoretico-politica della Repubblica (si vedano soprattutto, fra gli studi raccolti nel volume di F. Lisi, ed., The Ascent to the Good, Sankt Augustin, 2007, F. Calabi, L'analogia solare del VI libro della Repubblica; F. Ferrari, La potenza del 'Buono'; F. Lisi, The Form of the Good; L. Brisson, El sembrador divino (phutourgós); M. Abbate, Linterpretazione del Bene nella Dissertazione XI del Commento alla Repubblica di Proclo; e inoltre l'intero volume curato da D. Cairns, F.-G. Herrmann and T. Penner, eds., Pursuing the Good. Ethics and metaphysics in Plato's Republic, Edinburgh, 2007) o ancora come principio trascendente che costituisce il culmine della ricerca filosofica e della costruzione ontologica, o meta-ontologica, di Platone (penso particolarmente ai volumi di S. Roux, La recherche du principe chez Platon, Aristote et Plotin, Paris, 2004, e di S. Delcomminette, Le Philèbe de Platon. Introduction à l'agathologie platonicienne, Leiden-Boston, 2006) o infine dal punto di vista essenzialmente normativo della fondazione di una corretta pedagogia per i cittadini (questa la prospettiva adottata nel capitolo $\mathrm{X}$ del recente volume di $\mathrm{S}$.

PLATO, The electronic Journal of the International Plato Society, n 9, 2009.

http://gramata.univ-paris1.fr/Plato

(c) All rights of reproduction of any form reserved. 
Rosen, Plato’s Republic, New Haven \& London, 2008) o della costituzione etica e giuridica della città (secondo l'ottica assunta specialmente nel volume miscellaneo curato da D. Barbaric, hg., Platon über das Gute und die Gerechtigkeit, Würzburg, 2005), il dibattito sull'idea del bene appare tanto aperto e problematico da risultare irriducibile a qualunque semplificazione o schematizzazione che raccolga un accordo unanime, per quanto minimale, fra i commentatori e gli interpreti, al punto da indurre alcuni scholars a una sorta di inconfessata rinuncia (4).

Alla questione dei rapporti fra la teoria delle idee, la metodologia dialettica e la concezione platonica del linguaggio sono dedicati due studi di F. Mié: Dialéctica, predicación y metafísica en Platón, Cordoba, 2004, e Lenguaje, conocimiento y realitad en la teoría de las ideas de Platón, Cordoba, 2004. Il primo di essi si concentra soprattutto sulla teoria delle idee nei dialoghi di mezzo, dunque a partire dal Fedone, dal Menone e fino alla Repubblica. In tale ambito vengono presi in considerazione alcuni problemi classici: le origini della dialettica e la natura della conoscenza vera (pp. 21-62), la metafisica dei due mondi e i diversi gradi epistemologici corrispondenti alla scienza e all'opinione (pp. 63-91), la crisi onto-epistemologica che pare messa in scena nel Parmenide e la relativa difesa delle possibilità e delle condizioni operative della dialettica (pp. 93-185). Successivamente, Mié affronta la questione del rapporto fra l'onto-epistemologia eidetica e la sfera logico-linguistica, per stabilire le 'regole' del linguaggio e i suoi criteri di verità e correttezza, innanzitutto attraverso un serrato esame del Cratilo (pp. 187-229), quindi indagando approfonditamente la gnoseologia, che egli chiama 'anti-dialettica', del Teeteto (pp. 231-387). La seconda opera in esame prosegue e compie il percorso intrapreso nella prima, occupandosi dei dialoghi tardi, con particolare riguardo al Sofista. Dopo un capitolo iniziale che svolge una serie di utili considerazioni metodologiche (pp. 11-17), Mié si rivolge direttamente all'aporia del falso e del non essere nel Sofista (pp. 19-40), per ampliare di seguito l'orizzonte alle categorie generali dell'essere, dell'identico e del diverso, di cui traccia opportunamente le relazioni dialettiche (pp. 41-100) che

PLATO, The electronic Journal of the International Plato Society, n 9, 2009.

http://gramata.univ-paris1.fr/Plato

(c) All rights of reproduction of any form reserved. 
costituiscono il cuore della riflessione condotta dallo Straniero di Elea sui 'generi più grandi' e sulla loro koเvwvía (pp. 101-31). Nel capitolo seguente, viene sottoposto ad analisi il metodo dialettico nelle sue modalità diairetiche (pp. 13369) che, secondo Mié., conduce a considerare gli aspetti numerico-matematici dell'ontologia delle idee (pp. 171-213), specie rispetto alla dialettica dell'unità e della molteplicità o, in altri termini, del $\pi \dot{\varepsilon}$ pas e dell'ä $\pi \varepsilon ı \rho o v$, con ciò che ne deriva dal punto di vista della possibilità di composizione o di scomposizione delle idee, della loro derivazione meta-ontologica e della loro partecipazione da parte delle cose sensibili (pp. 215-54). L'ultima parte del volume contiene rispettivamente una valutazione teoreticamente impegnativa dell'ontologia platonica, nei suoi risvolti dinamici ed etici (pp. 255-92), con la concreta applicazione del principio universale del bene nella vita razionale e filosofica nel Filebo (pp. 293-329). Per quanto non particolarmente originale, l'approccio di Mié ha il merito di operare una ricognizione dettagliata del nesso 'conoscenza-linguaggio-realtà, che restituisce con chiarezza, anche se talvolta in modo poco problematico, le linee generali della riflessione platonica sulla funzione logico-semantica della sfera linguistica e sulla sua dipendenza dall'ambito ontologico delle idee, fornendo altresì, per questa via, un'illustrazione soddisfacente delle ragioni della centralità della dialettica nella filosofia di Platone, come metodo capace di ricondurre l'opzione discorsiva alla verità della conoscenza attraverso il necessario riferimento, comune al pensiero e al discorso, al valore normativo dell'intellegibile (5).

Tanto numerosi sono gli studi su questi aspetti della filosofia di Platone nei diversi suoi dialoghi singolarmente presi che sarebbe impossibile fornirne un esame compiuto e dettagliato (6).

PLATO, The electronic Journal of the International Plato Society, n 9, 2009.

http://gramata.univ-paris1.fr/Plato

(c) All rights of reproduction of any form reserved. 


\section{NOTE}

(1) Bisogna ricordare inoltre tre volumi recenti, di carattere introduttivo o manualistico, che presentano tuttavia non pochi spunti di interesse per le questioni esaminate. Il primo, in lingua tedesca: M. van Ackeren, hg., Platon verstehen.Themen und Perspektiven, Darmstadt, 2004: E. Heitsch, Frömmigkeit als Hilfe. Bemerkungen zum Euthyphron; D. Cürsgen, Platons Euthydem. Zum Verhältnis von Dialog. Logik und königlicher Kunst; E. Halper, Dialog und Argument in Platons Protagoras; M. Erler, 'Sokrates in der Höhle'. Argumente als affekstherapie im Gorgias und Phaidon; O.F. Summerell, Der Wollfaden der Liebe. Anmerkungen zu einem Motto in Platons Symposion; M. von Ackeren, Die Unterscheidung von Wissen und Meinung in Politeia $V$ und ibre praktische Bedeutung; B. Strobel, Attribute der Formen und die Form des Guten. Gerasimos Santas über die metaphysische Theorie des Guten in Platons Politeia; B. Effe, Platon und die Päderastie. Phaidros 256b-d und die platonische Eros-Theorie; D. Frede, Dialektik in Platons Spätdialogen; C. Horn, 'Niemand handelt freiwillig schlecht'. Moralischer Intellektualismus in Platons Nomoi?; J. Szaif, Die aletheia in Platons Tugenlehre; M. F. Meyer, Platon als Erfinder und Kritiker der Rhetorik; J. Hardy, Was wissen Sokrates und seine Gesprächtner? Überlegungen zu perfectem und menschlichem Wissen bei Platon; J. Halfwassen, Platons Metaphysik des Einen. Il secondo, in lingua francese: L. Brisson et F. Fronterotta, éds, Lire Platon, Paris, 2006: I Prédécesseurs et contextes historiques: M.-L. Desclos, Platon l'historien; L. Brisson et J.-F. Pradeau, Platon et les préplatoniciens; L.-A. Dorion, La figure paradoxale de Socrate dans les dialogues de Platon; A. Balansard, Les sophistes et les techniques; II La philosophie, les discours et les savoirs: C. Gill, Le dialogue platonicien; J.-F. Pradeau, Le bon usage du discours faux: les mythes; L. Brisson, La science et les savoirs; J.-F. Pradeau, Les divins gouvernants: la philosophie selon Platon; III Le sensible et l'intelligible: L. Brisson et A. Macé, Le monde et les corps;

PLATO, The electronic Journal of the International Plato Society, n 9, 2009. http://gramata.univ-paris1.fr/Plato (c) All rights of reproduction of any form reserved. 
F. Fronterotta, Qu'est-ce qu'une Forme pour Platon? Raisons et fonction de la théorie des intelligibles; F. Ferrari, Platon et la théorie des principes; IV Le corps et l'âme: L. Brisson, Le continuum de la vie chez Platon : des dieux aux plantes; F. Fronterotta, La réalité de l'âme: principe de vie, source de mouvement, sujet de connaissance; Y. Lafrance, La connaissance: science et opinion; $V$ Gouvernement de soi et gouvernement de la cité: A. Merker, «Nul n'est méchant de son plein gré»; L. Mouze, Éduquer l'humain en l'homme: l'auvre esthétique et politique du philosophe; M. Vegetti, Royauté et philosophie chez Platon; F. Lisi, La politique platonicienne: le gouvernement de la cité; VI La postérité: L. Brisson, L’histoire de l'Académie et la tradition platonicienne. Il terzo, in lingua inglese: G. Fine, ed., Oxford Handbook of Plato, Oxford, 2008: M. Schofield, Plato in His Time and Place; T.H. Irwin, The Platonic Corpus; M.M. McCabe, Plato's Ways of Writing; G.B. Matthews, The Epistemology and Metaphysics of Socrates; D. Devereux, Socratic Ethics and Moral Psychology; C.C.W. Taylor, Plato's Epistemology; V. Harte, Plato's Metaphysics; P. Crivelli, Plato's Philosophy of Language; H. Lorenz, Plato on the Soul; J. Annas, Plato's Ethics; R. Kraut, Plato on Love; C. Bobonich, Plato's Politics; R. Kamtekar, Plato on Education and Art; D. Scott, The Republic; S. Peterson, The Parmenides; M.K. Lee, The Theaetetus; L. Brown, The Sophist on Statements, Predication, and Falsehood; T. Johansen, The Timaeus on the Principles of Cosmology; C.C. Meinwald, The Philebus; C. Shields, Plato and Aristotle in the Academy; C. Brittain, Plato and Platonism.

(2) Si vedano solo, in tale direzione, M. Baltes, Zum Status der Ideen in Platons Frühdialogen Charmides, Euthydemos, Lysis, in Plato. Euthydemus, Lysis, Charmides. Proceedings of the V Symposium Platonicum, ed. by Th. Robinson \& L. Brisson, Sankt Augustin, 2000, pp. 317-23; F. Fronterotta, Methexis. La teoria platonica delle idee e la partecipazione delle cose empiriche. Dai dialoghi giovanili al Parmenide, Pisa, 2001; W.J. Prior, Socrates Metaphysician, 'Oxford Studies in

PLATO, The electronic Journal of the International Plato Society, n 9, 2009. http://gramata.univ-paris1.fr/Plato (c) All rights of reproduction of any form reserved. 
Ancient Philosophy', 27 (2004), pp. 1-14; G. Giannantoni, Dialogo socratico e nascita della dialettica nella filosofia di Platone, ed. B. Centrone, Napoli, 2005; e infine F. Fronterotta, The Development of Plato's theory of ideas and the Socratic question, in 'Oxford Studies in Ancient Philosophy', 32 (2007), pp. 37-62.

(3) Si vedano, di D. Wolfsdorf, gli articoli seguenti: Understanding the 'Whay is F' question", in 'Apeiron', 36 (2003), pp. 175-88; The Socratic fallacy and the epistemological priority of definitional knowledge, in 'Apeiron', 37 (2004), pp. 35 67; Interpreting Plato's early dialogues, in 'Oxford Studies in Ancient Philosophy', 27 (2004), pp. 15-40; Euthyphro 10a2-11b1: A study in Platonic metaphysics and its reception since 1960, in 'Apeiron', 38 (2005), pp. 1-71; Av́vaus in Laches, in 'Phoenix', 59 (2005), pp. 324-47; Aïtov and aitía in Plato, in 'Ancient Philosophy', 25 (2005), pp. 341-48; Hippias Major 301b2-c2: Plato's critique of corporeal conception of Forms and of the Form-Participant relation, in 'Apeiron', 39 (2006), pp. 221-56.

(4) Abbastanza stupefacente, infatti, la pressoché completa assenza di riferimenti al problema dell'idea del bene in G. Santas, ed., The Blackwell Guide to Plato's Republic, Blackwell Publishing, 2006, in cui nessun saggio è espressamente dedicato alla questione; anche i contributi apparentemente pertinenti di M. Anagnostopoulos, The Divided Soul and the Desire for the Good in Plato's Republic, di M.T. Ferejohn, Knowledge, Recollection and the Forms in Republic VII e di T. Penner, Plato's Theory of Forms in the Republic, rimangono su questo punto estremamente generici e superficiali.

(5) Ricordo inoltre, su questo tema, lo studio di E. Heitsch, Platon und die Anfänge seines dialektischen Philosophierens, Göttingen, 2004. Piuttosto agli aspetti formali e stilistici della struttura narrativa del dialogo platonico è invece dedicato l'utile lavoro di R. Geiger, Dialektische Tugenden. Untersuchungen zur

PLATO, The electronic Journal of the International Plato Society, n 9, 2009. http://gramata.univ-paris1.fr/Plato (c) All rights of reproduction of any form reserved. 
Gesprächsform in den Platonischen Dialogen, Paderborn, 2006.

(6) Mi limito perciò a fornire un elenco, non esaustivo, di questi lavori. Sul Cratilo: A. Eckl, Sprache und Logik bei Platon, I. Logos, Name und Sache im Kratylos, Würzburg, 2003; sul Menone: M. Erler \& L. Brisson, eds., Gorgias Menon. Selected Papers from the seventh Symposium Platonicum, Sankt Augustin, 2007; sulla Repubblica: M. Dixsaut, éd., Études sur la République, 2 voll., Paris, 2005; sul Teeteto: P. Stern, Knowledge and politics in Plato's Theaetetus, Cambridge, 2008; sul Parmenide: Plato's Parmenides. Proceedings of the fourth Symposium Platonicum Pragense, ed. by A. Harák and Filip Karfík, Prague, 2005; S. Rickless, Plato's Forms in transition. A reading of the Parmenides, Cambridge, 2007; sul Sofista: N. Notomi, The Unity of Plato's Sophist, Cambridge, 1999; C. Ziermann, Platons negative Dialektik: eine Untersuchung des Dialoge Sophistes und Parmenides, Würzburg, 2004; M. Migliori, Il Sofista di Platone. Valore e limiti dell'ontologia, Brescia 2006 (trad. ingl.: Plato's Sophist. Value and Limitation of Ontology, Sankt Augustin, 2007); D: Ambuel, Image and Paradigm in Plato's Sophist, Las Vegas, 2007; sul Filebo: Schmidt-Wilborg, Dialektik in Platons Philebos, Tübingen, 2005. Segnalo a parte, per il suo carattere davvero monumentale, la traduzione italiana commentata della Repubblica, con numerosi saggi di commento sui diversi aspetti e problemi posti dalla lettura del dialogo, a cura di M. Vegetti, 7 voll., Napoli, 1998-2007; a questa opera, e in generale agli studi recenti sulla Repubblica, dedicherò un contributo in uno dei prossimi numeri del 'Journal of the History of Philosophy'. Ricordo infine, per il loro carattere 'misto' nell'approccio alla problematica ontologica in Platone, il volumetto miscellaneo curato da M. Bianchetti \& E.S. Storace, Platone e l'ontologia. Il Parmenide e il Sofista, Milano, 2004 (con saggi di E. Berti, G. Casertano, F. Fronterotta, M. Migliori, C. Sini, M. Vegetti e V. Vitiello), e lo studio di T.-B. Yang, Platon in der philosophischen Geschichte des Problems des Nichts, Würzburg, 2005.

PLATO, The electronic Journal of the International Plato Society, n 9, 2009. http://gramata.univ-paris1.fr/Plato (c) All rights of reproduction of any form reserved. 